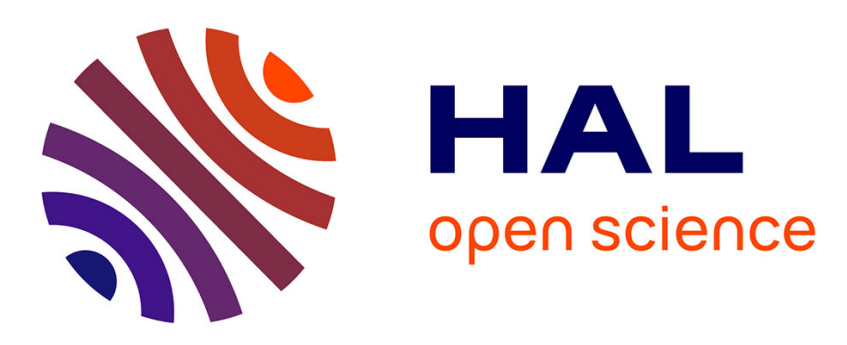

\title{
SDN-based RAN Protection Solution for 5G, an Experimental Approach
}

\author{
Minqi Wang, Gael Simon, Isabel Amigo, Luiz Anet Neto, Loutfi Nuaymi, \\ Philippe Chanclou
}

\section{- To cite this version:}

Minqi Wang, Gael Simon, Isabel Amigo, Luiz Anet Neto, Loutfi Nuaymi, et al.. SDN-based RAN Protection Solution for 5G, an Experimental Approach. ONDM 2021: International Conference on Optical Network Design and Modeling, Jun 2021, Gothenburg, Sweden. pp.1-6, 10.23919/ONDM51796.2021.9492454 . hal-03513270

\section{HAL Id: hal-03513270 https://imt-atlantique.hal.science/hal-03513270}

Submitted on 23 Feb 2022

HAL is a multi-disciplinary open access archive for the deposit and dissemination of scientific research documents, whether they are published or not. The documents may come from teaching and research institutions in France or abroad, or from public or private research centers.
L'archive ouverte pluridisciplinaire HAL, est destinée au dépôt et à la diffusion de documents scientifiques de niveau recherche, publiés ou non, émanant des établissements d'enseignement et de recherche français ou étrangers, des laboratoires publics ou privés. 


\title{
SDN-based RAN Protection Solution for 5G, an Experimental Approach
}

\author{
Minqi Wang ${ }^{(1)(2)}$, Gaël Simon ${ }^{(1)}$, Isabel Amigo ${ }^{(2)}$, Luiz Anet Neto ${ }^{(2)}$, Loutfi Nuaymi ${ }^{(2)}$, Philippe Chanclou ${ }^{(1)}$ \\ (1) Orange Labs, 2 Avenue Pierre Marzin, 22300 Lannion, France \\ (2) IMT Atlantique, 665 Avenue du Technopôle, 29200 Plouzané, France \\ minqi.wang@orange.com
}

\begin{abstract}
In this paper, we propose a Software Defined Network-based (SDN) Passive Optical Network (PON) Radio Access Networks (RAN) protection scheme. We experimentally assess the performance by implementing it through a testbed including an SDN controller, Small form-factor pluggable transceiver PON (SFP+/PON), a layer 2 switch, and several SFP+ Point-to-Point (SFP+/PtP) in lieu of a traditional OLT chassis. We compared our proposition with a PtP scheme based on legacy layer 2 redundancy protocol solution with respect to different metrics such as recovery time, latency, power consumption, CAPEX and flexibility/scalability. We show that a trade-off exists among those metrics and that while the SDN-based solution is outperformed by the legacy-based one in terms of recovery time, the proposed solution still provides a remarkable improvement with respect to nowadays practices in protection, while allowing for cost and energy reduction. Such solution scheme can thus be used as a protection scheme for non-time-critical services.
\end{abstract}

Keywords-Access Networks, RAN, SDN, 5G, PON, Protection

\section{INTRODUCTION}

5G demands for bandwidth, latency and availability needs for different services are vaster from the core network to end users compared to legacy mobile generations. This requires a technological revolution and could force mobile operators to completely upgrade their network architectures including the optical access network as well.

Availability is recurrently ensured using mesh and ring topologies for core and transport/aggregation optical transport networks respectively. The former approach is starting to be more commonly seen in wireless access networks as well. Concerning the last mile of fiber-based transport, the International Telecommunication Union (ITU) has listed the different considerations of Passive Optical Network (PON) protection on fixed access networks [1]. However, link protection in wireline access is still relatively rudimentary and will have to evolve in order to match the needs of next generation mobile services.

Indeed, radio network functions are implemented in different equipment at the Central Office (CO), antenna sites and eventually at intermediary locations depending on the Radio Access Network (RAN) functional split chosen by the operator [2]. The risk of unavailability comes mainly from the RAN connectivity: nowadays, $\mathrm{CO}$ equipment, which can be either an

978-3-903176-33-1 (C) 2021 IFIP
Optical Line Termination (OLT) or a layer $2 / 3$ device such as a switch or a router, is connected to each cell-site gateway at the antenna premises through a single fiber (bidirectional transmission) by means of a Point-to-Point (PtP) link. A failure can appear when the equipment in the $\mathrm{CO}$ dysfunctions or if the single fiber is cut between the $\mathrm{CO}$ and the antenna site. Starting from a complaint of the antenna mobile operator, a technical intervention from an infrastructure operator then takes place and the guarantee of recovery time is usually in the order of hours depending on the service level agreement (SLA) negotiated between the infrastructure operator and the mobile operator. However, 5G requires very strict (low) recovery times. For instance, the Open-RAN initiative defined $50 \mathrm{~ms}$ as the $\mathrm{x}$-hauling recovery time for data plane regardless of the type of $5 \mathrm{G}$ service [3]. Moreover, the access networks availability intended for $5 \mathrm{G}$ is to reach $99,999 \%$ or more depending on certain services according to the 3GPP [4]. This means that a protection link should be established with independent path and an autorecovery mechanism should be applied between the main link and the protection link [5].

At the same time, virtualization technologies have achieved a considerable progress from core to access optical networks. Many projects are carried out fostering technical innovations: Open Networking Foundation (ONF) has launched the Central Office Re-architected as a Datacenter (CORD) project [6] several years ago with the goal of virtualizing the access and backhaul networks and leveraging recent practices related to technologies such as Software Defined Network (SDN), Network Functions Virtualization (NFV) and Cloud infrastructures. Mobile-CORD and Residential-CORD were initially included in CORD. Then, the projects Converged Multi-Access and Core (COMEC) and SDN Enabled Broadband Access (SEBA) were derived from $\mathrm{M}-\mathrm{CORD}$ and R-CORD with SEBA focusing on SDN solutions over access and aggregation network with white box OLT hardware. The term "white box" refers to solutions that allow for the decoupling of hardware and software in network equipment. This offers the advantage of enabling a much more flexible, vendor-rich and cost-efficient eco-system. Being part of R-CORD, the virtual OLT Hardware Abstraction (VOLTHA) project [7] is carried out to virtually reconsider the whole OLT as an Open Flow switch [8], from the SDN controller point of view, thanks to the abstraction layer. In order to do so, the data plane and the control plane are separated and their SDN controller (e.g. SEBA) runs based on the OLT abstraction model. 


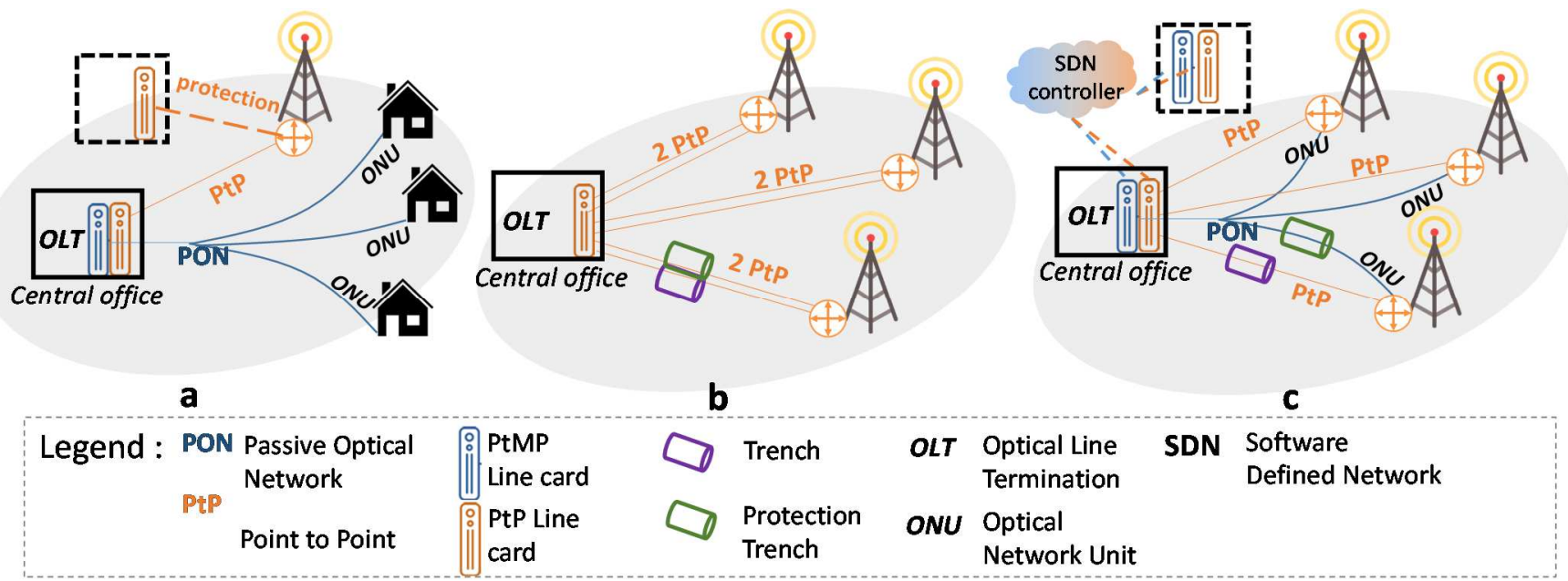

Fig. 1: a) Protection architecture b) PtP protection solution c) PON protection solution.

In parallel, NTT carried out the Flexible Access System Architecture (FASA) project [9] to virtualize the functions of OLT in a general server (e.g. x86). The concept is to compose flexible, modularized functions on commodity hardware. In 2019, a software-based OLT for 10G-EPON upstream Layer Processing was realized on a general server [10]. Recently, their work was merged to project VOLTHA and [11] shows a successful implementation of a modular OLT with Openflow switch.

Other studies are now focusing on the new use cases and algorithms of Dynamic Bandwidth Allocation (DBA) to share the OLT through multi-tenancy approaches and increase PON performance for instance in terms of latency [12]. Driven by all research contributions and standardization bodies' effort on access network's virtualization, the telecommunication equipment today embeds more and more SDN/NFV functionalities such as YANG models [13] and NETCONF/RESTCONF [14] protocols.

In this context, we aim to apply a pragmatic SDN solution for operators to benefit directly from the phase of transition from legacy telecommunication equipment to fully virtualized setup. Comparing to public $5 \mathrm{G}$ networks, non-public network such as industrial and campus applications might have different and broader requirements. Requirements concern SLAs, traffic profiles and the needs in terms of automation and the control plane in general [15]. Thus, developing SDN/NFV technologies for non-public network's protection could be more useful at first place. A protection link from the second $\mathrm{CO}$ using a PtP topology, as shown in Fig. 1a, can cover outages from both the $\mathrm{CO}$ and the physical fiber link but this solution would need higher capital expenditures (CAPEX) upon deployment and also higher operational expenditures (OPEX) to maintain the backup site at a different geographical location.

The scenarios we propose in this paper are based on the generic architecture used for macro-cell in France and tested in laboratory. We replace a legacy OLT chassis by a layer 2 switch with smart Small form-factor pluggable transceiver PONs (SFP+/PON) to benefit from the numerous advantages brought by the virtualization tendencies in the optical access. We introduce an SDN-oriented self-healing solution based on PON technology and we compare it with the conventionally PtP layer 2 solution.

We first introduce in detail the proposition and the use case of our work in Section II. We then describe the experimental setup in Section III and the corresponding result in Section IV. In Section V, we elaborate a mathematical model to estimate the CAPEX and energy consumption for each solution, depending to the size of the CO. Finally we discuss and conclude this work in Section VI.

\section{PROPOSAL}

Fig. $1 \mathrm{a}$ and $\mathrm{b}$ show typical examples of protection for $\mathrm{a}$ mobile access connectivity network. Fig. 1a depicts the most complete and expensive approach, where both protection central office and link are used. Notice that in the case of a PON based approach, the same OLT can be used for both PtP and PtMP applications and this is typically done by means of separated Optical Distribution Networks (ODNs) and different line cards, which are represented with different colors in Fig. 1a. In Fig. $1 \mathrm{~b}$ a less effective but more interesting approach in terms of CAPEX is shown, this time with only one central office equipment for both the protection and normal links but still with separated fibers, in different trenches. Fig. 1b would allow protection in case of a fiber cut but not in case of an equipment dysfunction, as opposed to Fig. 1a. Fig. 1c shows the details of the implementation for our SDN-based PON protection solution that allows for a simpler yet more cost-effective protection solution by using one single $\mathrm{CO}$ in a high density industry or campus area and we evaluate their pros and cons.

Fig. 2 shows the proposed architecture of the SDN-based OLT for PON protection. The switch can host both PtP links (for regular connections), with $\mathrm{SFP}+\mathrm{PtP}$ transceivers, and $\mathrm{PON}$ links (in blue on Fig. 2) with SFP+/PON transceivers. In the case of the PON links, we also replace a conventional Optical Network Unit (ONU) by an SFP+/ONU. An SDN controller is developed and has an overview vision of the switch, the $\mathrm{SFP}+\mathrm{PON}$ and attached SFP+/ONU thanks to the adapter developed in our controller. The SDN controller controls the $\mathrm{SFP}+/ \mathrm{PON}$ and $\mathrm{SFP}+/ \mathrm{ONU}$ by ONU Management and Control 


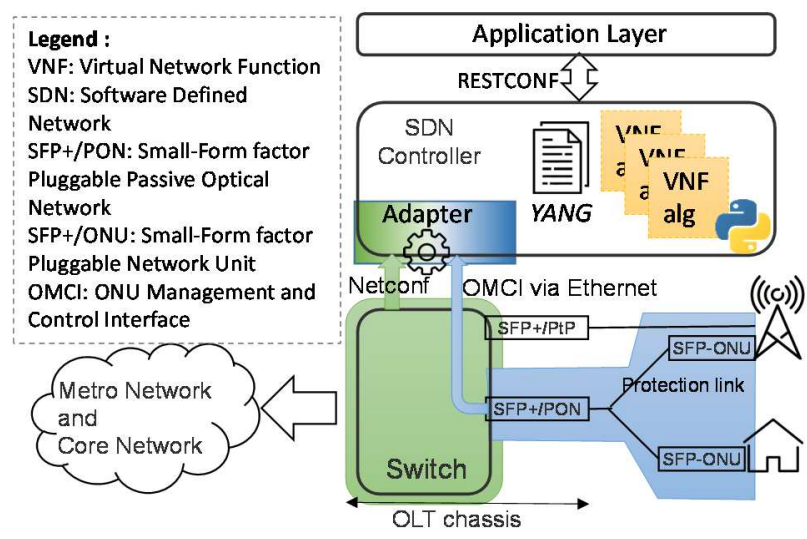

Fig. 2: Architecture of switch-SFP+/PON and SFP+/PtP based OLT with SDN controller

Interface (OMCI) over Ethernet, and controls the switch by using NETCONF protocol.

Virtual Network Functions (VNF) may be developed for access network automation as well. In our previous work, a dynamic management of backplane ports of OLT according to the PtP ports and PON ports' real time throughput has been proposed [16]. A YANG model was implemented and the data in YANG model is refreshed automatically thanks to the connection existing between the SDN controller and the physical equipment. A web application could consult configuration data in YANG and launch different VNF algorithms to fit the needs of operators. In this work, we added another functionality to our previous architecture, since a different VNF is introduced to deal with the $5 \mathrm{G}$ self-healing problem. This functionality is used for the PON protection solution on the experimental setup described in this work.

In this paper, we focus on the transport of mobile backhaul or midhaul traffic. Our proposal is based on the fact that an OLT is an aggregation node that traditionally consists of different embedded cards. Line cards for residential services use PON technology with PtMP architecture and the line cards for mobile services or enterprise services use PtP architecture.

We first implemented what we call a legacy solution which consists in establishing a second PtP link as a protection link for each antenna site (Fig. $1 \mathrm{~b}$ ). In this solution, two ports are required on the $\mathrm{CO}$ equipment. The second proposition is our SDN-based solution which consists in using a single PON tree (PtMP) as the protection link for all antenna sites (Fig. 1c). Since the link unavailability for $5 \mathrm{G}$ is in the orders of minutes per year [5], without counting OLT failures, the risk is low for several links connecting different antenna sites to experience a failure at the same time. Indeed, assuming only one antenna failure at a time per PON tree, the PON link would virtually behave as a PtP connection, while mutualizing the deployed fiber infrastructure. We experimentally demonstrate the scenario where the main PtP link is disrupted and the data plane recovery is done automatically through the protection link. Once the PtP link is reestablished, the traffic is sent back to the original path automatically.

In the next section, our detailed experimental setup will be listed. Based on this setup, we assess our solution according to the following factors: needed recovery time, latency, availability, energy consumption, infrastructure cost and flexibility /scalability.

\section{EXPERIMENTAL SETUP}

The benchmark setup in Fig. 3a is a simple PtP protection link that consists in conventional switches and SFP+/PtP. Our proposed setup in Fig. $3 \mathrm{~b}$ uses a single $\mathrm{PON}$ for all protection links with 10G-EPON technology. Here, the programmable NETCONF/Yang switch [13] together with the intelligent $\mathrm{SFP}+/ \mathrm{PON}$ plays the role of the OLT in the central office (CO) [17]. SFP+/ONUs are chosen for PON protection solution since such a small and flexible module can be inserted directly into the pre-existing switch near the antenna site. Thanks to the $\mathrm{SFP}+/ \mathrm{PON}$ and SFP+/ONUs modules, no extra equipment deployment is needed at $\mathrm{CO}$ or at cell site gateway. Furthermore, $\mathrm{SFP}+/ \mathrm{ONUs}$ are more economical compared to legacy ONUs. An Ethernet traffic generator/analyzer sends/receives 2x10Gbps traffic to simulate $10 \mathrm{Gbps}$ traffic for two antenna sites.

As previously mentioned, the protection link is necessary for rapidly recovering the data plane traffic. Some features already exist on the switch for a redundant solution by configuring an additional port as the protection port: if the primary link is down, the protection port takes over automatically the traffic from primary port. For doing so, both
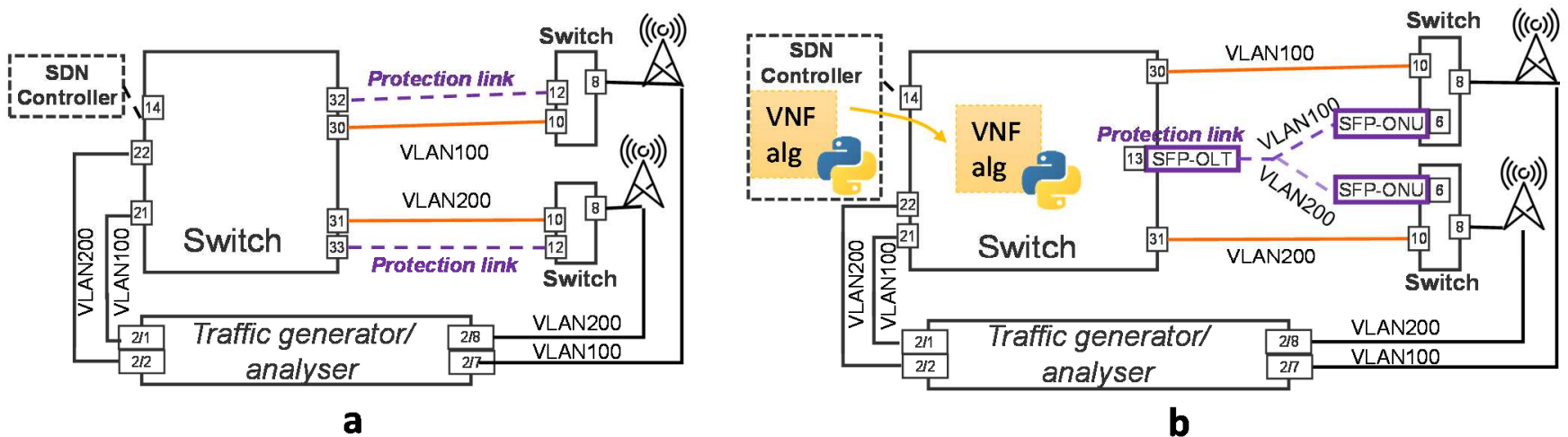

Legend : VNF: Virtual Network Function SDN: Software Defined Network SFP: Small-Form factor Pluggable

Fig. 3: Experimental setup: a) PtP protection solution b) PON protection solution 

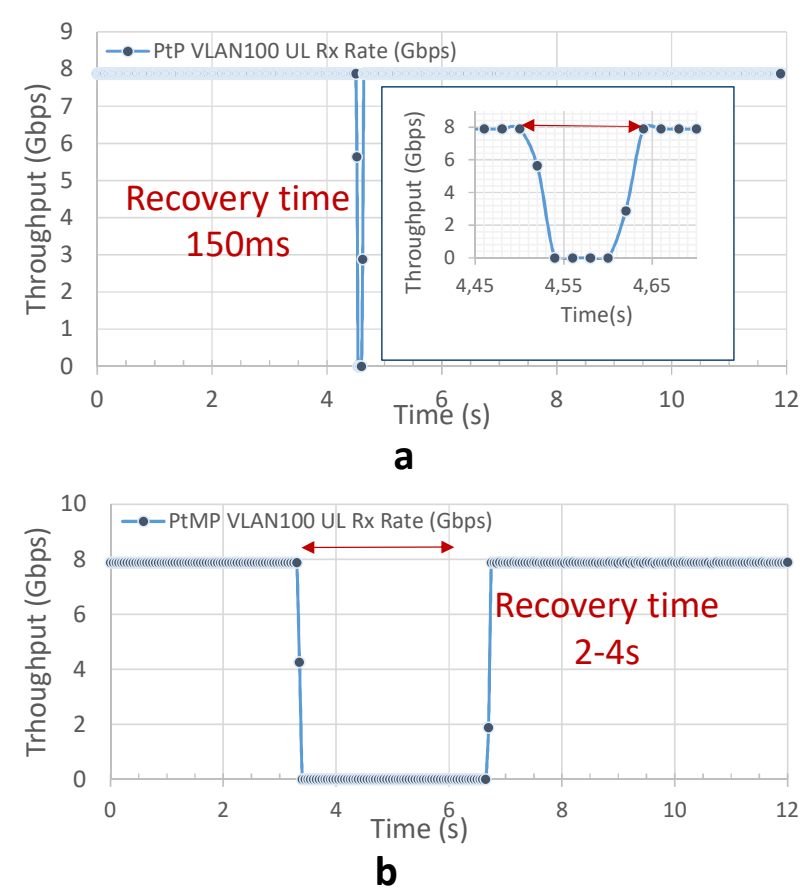

Fig. 4: Recovery time experimental results for PtP and PON protection solutions

primary port and protection port should have exactly the same configuration. Also, in this configuration, one protection port can only to backup one primary port. Therefore, features such as Redundant Trunk Groups are suitable for benchmark PtP protection solution in Fig. 3a where each PtP primary link has its own secondary link as backup. For the PON protection scenario, only one port of the CO side hosting a $\mathrm{SFP}+\mathrm{PON}$ is needed to connect and backup all antenna sites. In our solution, an algorithm is implemented to monitor the traffic in real time, detecting an outage of the primary links and redirecting the traffic to the protection link. This algorithm whthin the SDN controller named VNF in Fig. 2 communicates with the switch using NETCONF protocol. This lightweight VNF shown in Fig. $3 \mathrm{~b}$ could be placed flexibly in a generic server such as in a distant SDN controller node or in the CO. In our implementation, in order to compare more precisely the date recovery time between two methods, we optimized the transmission delay between the $\mathrm{CO}$ and the distant generic server and implemented the VNF algorithm in the computing modules of the switch hosting the $\mathrm{SFP}+/ \mathrm{PON}$.

\section{EXPERIMENTAL RESULTS}

Thanks to a traffic analyzer, we can determine the recovery time for each solution according to the throughput at the receiver port (Fig. 4). Table 1 shows the criteria for current deployment, including the goal of recovery time as well. Both solutions provide remarkable improvement compared to today's sites that need technical intervention although none of them respects the $50 \mathrm{~ms}$ imposed for future usages. The PtP protection solution needs only $150 \mathrm{~ms}$. For the PON solution, NETCONF is the communication protocol used between the physical equipment (switch) and our VNF algorithm. A commit phase, intrinsic to the NETCONF protocol, takes at least $1.5 \mathrm{~s}$ which causes a $2 \mathrm{~s}-$
$4 \mathrm{~s}$ recovery time as result. This could be minimized by using other protocols [14] [18]. The maximum latency of the uplink (UL) and downlink (DL) during the experimental scenario are presented in Table 1. Both solutions have a reasonable latency and they fit well within $1 \mathrm{~ms}$, which corresponds to the most strict latency goal of backhaul and midhaul. Please note that the UL latency of PON is much larger than the PtP link. This is related to Time division multiple access (TDMA) technology applied on PtMP architecture and the latency depends on performance of DBA and numbers of ONUs as well. Times of failures are calculated for both solutions and the availability is taken from [5] and are used to provide the last row in Table 1.

Table 1: Comparison of experimental results for two protection solutions

\begin{tabular}{|l|l|l|l|l|}
\hline $\begin{array}{l}\text { Compare } \\
\text { items }\end{array}$ & $\begin{array}{l}\text { Current } \\
\text { status }\end{array}$ & Goal & $\begin{array}{l}\text { PtP } \\
\text { solution }\end{array}$ & $\begin{array}{l}\text { PON } \\
\text { solution }\end{array}$ \\
\hline $\begin{array}{l}\text { Data plane } \\
\text { Recovery } \\
\text { time }\end{array}$ & $1-2 \mathrm{~h}$ & $50 \mathrm{~ms}$ & $150 \mathrm{~ms}$ & $2-4 \mathrm{~s}$ \\
\hline $\begin{array}{l}\text { Latency } \\
\text { (ms) }\end{array}$ & $\begin{array}{l}\text { Backhaul } \\
50\end{array}$ & $\begin{array}{l}\text { Backhaul } \\
1-50 \\
\text { Midhaul } \\
1,5-10\end{array}$ & $\begin{array}{l}\text { UL 0,008 } \\
\text { DL 0,009 }\end{array}$ & $\begin{array}{l}\text { UL 0,771 } \\
\text { DL 0,016 }\end{array}$ \\
\hline $\begin{array}{l}\text { Availabilit } \\
\text { y/year }\end{array}$ & $\begin{array}{l}99,9 \% \\
=8,76 \mathrm{~h}\end{array}$ & $\begin{array}{l}99,999 \% \\
=5 \mathrm{~min}\end{array}$ & $\begin{array}{l}5 \mathrm{~min} / 150 \\
\mathrm{~ms}=2000 \\
\text { failures }\end{array}$ & $\begin{array}{l}5 \mathrm{~min} / 3 \mathrm{~s}= \\
100 \\
\text { failures }\end{array}$ \\
\hline
\end{tabular}

\section{COST AND ENERGY CONSUMPTION COMPARAISON}

Even if the SDN solution proposed here provides relatively fast recovery times (see Fig. 4 in previous section), it is less performing than a PtP-based layer 2 solution. However, the main disadvantages of the PtP-based solution is that it is realized by doubling the number of access nodes for mobile connectivity. Furthermore, the backup port in the OLTs could not be used for other purposes since the RTG mechanism binds the primary port to the protection port. On the contrary, our SDN-oriented PON solution offers a flexible VNF function from SDN server to switch with $\mathrm{SFP}+\mathrm{PON}$ in the CO. As a result, only one additional port is needed in the OLT to protect all mobile PtP links from a $\mathrm{CO}$.

Considering that the CAPEX and energy consumption reduction are essential for telecommunication operators, we propose a model to estimate and compare the cost and energy consumption of two protection architectures. This model is based on the number of PtP ports used in one $\mathrm{CO}$ and takes into consideration all equipment used at the $\mathrm{CO}$ since we always need two ports at antenna site so the cost is considered as the same whatever the protection solution is used.

Assuming that $N$ antenna sites are connected to a single CO by a PtP link, then $N$ ports are needed originally at $\mathrm{CO}$ without any protection procedure. In case of a PtP-based protection solution, each PtP connection will have a second PtP link as protection link, so twice the number of ports are required $(2 N)$. In the proposed PON protection scenario, assuming the PON split ratio is 64 and $\mathrm{N}$ is less than 64, this number is $N+1$ because 
only one additional port in the $\mathrm{CO}$ is needed: all antenna sites around this $\mathrm{CO}$ share a single PON architecture. Another important advantage of our solution is that the OLT in the CO is either a conventional OLT chassis with line cards or a layer 2 switch with smart SFP+/PON. When the maximum number of ports per board (line card for standard OLTs or switch for layer 2 based OLT) is achieved, new boards need to be added which introduces necessarily extra expenses. Assuming $M$ ports (according to a legacy OLT $M=8$ ) by board, according to a total number of ports previously mentioned ( $2 N$ for PtP, $N+1$ for PON), the PtP solution needs $[2 N / 87$ boards whereas the PON needs $\lceil(N+1) / 8\rceil$ boards (here, $\lceil x\rceil$ stands for the ceiling operation on number of $x$ to the closes integer).

In addition, the cost and energy consumption for SFP+/PON and SFP+/PtP at $\mathrm{CO}$ are not negligible. The notations and their values normalized in Table 2 will help us express the cost and energy consumption estimations by using L2/3 switch as reference cost and consumption. The normalized values are calculated based on real values of our testbed in laboratory.

Table 2: Normalized cost and consumption of each component

\begin{tabular}{|l|l|l|l|}
\hline Unit Items & SFP+/PON & SFP+/PtP & Switch \\
\hline Cost $(\$)$ & $\mathrm{n}$ & $\mathrm{m}$ & $\mathrm{q}$ \\
\hline Normalized Cost & 1 & 0.025 & 1 \\
\hline $\begin{array}{l}\text { Energy } \\
\text { Consumption(W } \\
)\end{array}$ & $\mathrm{b}$ & $\mathrm{a}$ & $\mathrm{e}$ \\
\hline $\begin{array}{l}\text { Normalized } \\
\text { Consumption }\end{array}$ & 0.02 & 0.0067 & 1 \\
\hline
\end{tabular}

For example, the total cost in the $\mathrm{CO}$ with protection is the product of the cost of transceivers $m$ by the number of antenna sites $N$, added to the cost of the switch $q$ multiplied by the required number of boards, which is $/ N / M]$. The formula is provided by Equation (1). More generally, the functions of total cost and total energy consumption are listed below.

Total Cost without Protection $=m N+q\left\lceil\frac{N}{M}\right\rceil$

Total Cost with PtP Protection $=2 m N+q\left\lceil\frac{2 N}{M}\right\rceil$

Total Cost with PON Protection

$$
=m N+q\left\lceil\frac{N}{M}\right\rceil+n
$$

Energy calculation is pretty similar to the cost calculation, except that $a$ represents the energy consumption of an SFP $+/ \mathrm{PtP}$, $b$ for the energy consumption of an $\mathrm{SFP}+/ \mathrm{PON}$ and $e$ the energy consumption of a switch.

Total Energy Consumption without Protection

$$
=a N+e\left\lceil\frac{N}{M}\right\rceil
$$

Total Energy Consumption with PtP Protection

$$
=2 a N+e\left\lceil\frac{2 N}{M}\right\rceil+N a
$$

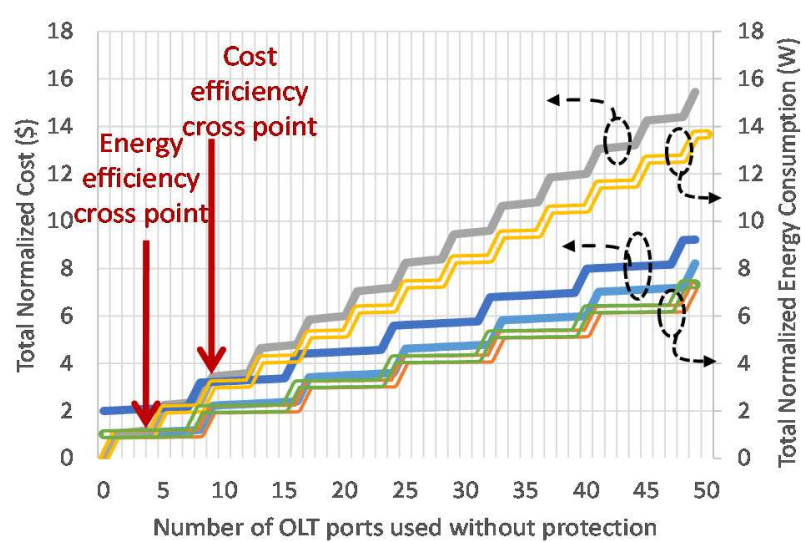

$$
\begin{aligned}
& \text { Cost without protection } \\
& \text { Cost with PtP protection } \\
& \text { Cost with PON protection } \\
& \text { Energy consumption without protection } \\
& \text { Energy consumption with PtP protection } \\
& \text { Energy consumption with PON protection }
\end{aligned}
$$

Fig. 5: Cost and energy consumption comparison between PtP and $\mathrm{PON}$ protection solution

Total Energy Consumption with PON Protection

$$
=a N+e\left\lceil\frac{N}{M}\right\rceil+b
$$

Fig. 5 shows all functions above with number of OLT ports without protection $(N)$ varying from 0 to 50 . As we can see in Fig. 5, if the numbers of antenna linked to a $\mathrm{CO}$ is lower than 5, the cost of PtP protection is cheaper than PON solution. Starting from $N=5$ until the cost efficiency cross point at $N=8$, the two solutions have a similar cost. Then, PON protection provides significantly better results than the PtP solution in cost after the cross point of $N=8$.

As for the energy consumption, the PON solution performs better than the PtP solution starting from 4 antenna sites connected to a $\mathrm{CO}$ according to Fig. 5. Furthermore, since our solution disables the SFP+/PON and ONU when those are not used (those are energy-saving capable modules), energy consumption is practically the same as the one where no protection at all is used.

\section{DISCUSSION AND CONCLUSION}

In this paper we have experimentally compared an SDNoriented PON and a layer $2 \mathrm{PtP}$ protection scheme for next generation fixed access mobile site connectivity in terms of recovery time and latency. Both solutions demonstrate a remarkable progress compared to today's recovery time by a technical intervention, since they allow for only a few seconds of service interruption compared to today's hour's long reaction times. Nevertheless, none of them fits in the 50ms long O-RAN Alliance requirements yet.

Cost and energy consumption for both protection solutions compared to the existing architecture are essential information for telecommunication operators to plan their deployment. In high density areas in France, a single CO could have more than 10 macro sites connected to it. Fig. 6 shows the COs and associated antenna repartition in Paris, France, based on Orange's data from 2019 (4G sites)[22]. The red links show 


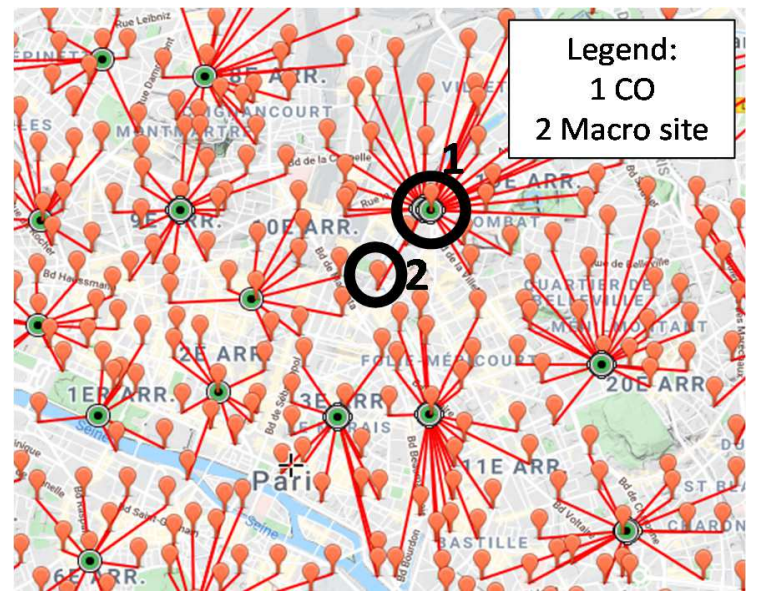

Fig. 6: $\mathrm{CO}$ and macro antenna site distribution in Paris

logical fiber connections from each $\mathrm{CO}$ to the antenna sites. With the deployment of $5 \mathrm{G}$ antennas, the number of macro sites will potentially increase, to allow high performance transmissions with seamless coverage.

In this sense, we performed calculations to quantify the CAPEX and OPEX in CO and compared precisely each proposition. The proposed PON allows for energy savings compared to PtP solution and also enables CAPEX reduction if there is more than 9 antennas' link connected to one single $\mathrm{CO}$, which could be very likely to happen according to our field data. As a result, the proposed PON protection solution is a pertinent architecture in dense areas. Besides, using a PON solution in dense areas would allow saving space in the COs as compared to the PtP backup solution, which doubles all needed infrastructure including the boards in COs.

The civil construction costs related to the trenching of the protection path from the $\mathrm{CO}$ to antenna sites were not considered because of the complexity of calculation in different scenarios (cities, mildly dense zones, green-field areas, etc). Yet, it's not hard to estimate that the PON could save money since a single trench is shared at the PON feeder segment. Another possibility resides in using more than one PON tree for protection services, in which case each PON tree has less antenna sites so that a higher quality of services can be assured if more than one site needs to use the protection link.

Finally, even though the SDN approach with PON solution is less performing in term of recovery time, its topology is much more advantageous in terms of energy consumption and infrastructure cost. Moreover, since the legacy OLT chassis is replaced by a switch and a modular SFP+/PON, the VNF function could take into consideration the whole OLT including the backplane of the switch and the PtP and PON connections. This enables a next step towards an evolution in terms of flexibility and scalability in optical access networks.

\section{ACKNOWLEDGMENT}

This work was supported by the European H2020-ICT-2019 project 5G-COMPLETE and H2020-ICT52-2020 project MARSAL.

\section{REFERENCES}

[1] Passive optical network protection considerations. ITU T-REC-G.Sup51. Jun. 2017.

[2] L Larsen, A Checko, and $\mathrm{H}$ Christiansen. A survey of the functional splits proposed for $5 \mathrm{G}$ mobile crosshaul networks. IEEE Communications Surveys \& Tutorials, 21(1):146-172, 2018.

[3] Xhaul transport requirements. Technical Report ORANWG9.Transport.0-v00.12 TS, 2020.

[4] 3GPP TS 22.261 Service requirements for the 5G system. Technical Report, V16.4.0, 062018.

[5] P Chanclou et al. Workshop: Converged 5G and heterogeneous services access networks: How to achieve ultra-low latency and high reliability? Optical Fiber Communication Conference (OFC), 2020.

[6] L. Peterson et al., "Central office re-architected as a data center," IEEE Commun. Mag., vol. 54, no. 10, pp. 96-101, Oct. 2016, doi: 10.1109/MCOM.2016.7588276.

[7] Open Networking Foundation. (2020). VOLTHA, [Online]. Available: https://opennetworking.org/voltha/

[8] OpenFlow Switch Specification. Technical Report version1.5.1 TS-025, March 2015.

[9] FASA®: New Access System Architecture. NTT, [Online]. Available: https://www.ntt-review.jp/archive/ntttechnical.php?contents= ntr201906fa2.html

[10] T. Suzuki, S.-Y. Kim, J. Kani, and J. Terada, "Software Implementation of 10G-EPON Upstream Physical-Layer Processing for Flexible Access Systems," J. Lightwave Technol., vol. 37, no. 6, pp. 1631-1637, Mar. 2019, doi: 10.1109/JLT.2018.2883912.

[11] K. Nishimoto, K. Asaka, J. Kani, and J. Terada, "Automated provisioning method for a modular PON-OLT device toward Plug Provision," IEEE/OSA Journal of Optical Communications and Networking, vol. 12, no. 9, pp. D9-D16, Sep. 2020.

[12] F. Slyne et al., "Experimental Demonstration of multiple Disaggregated OLTs with Virtualised Multi Tenant DBA, over General Purpose Processor," in Optical Fiber Communication Conference (OFC) 2020, San Diego, California, 2020.

[13] M. Bjorklund, Ed. YANG - a data modeling language for the network configuration protocol (NETCONF). (RFC6020), 102010.

[14] RFC 8040 - RESTCONF Protocol, 2017.

[15] 5G industry campus network deployment guideline. GSMA White paper V1.0, 112020.

[16] M. Wang, G. Simon, L. A. Neto, I. Amigo, L. Nuaymi and P. Chanclou, "Dynamic Traffic Management of OLT Backhaul/Service Ports with SDN Controller," 2020 European Conference on Optical Communications (ECOC), Brussels, Belgium, 2020.

[17] G Simon et al. FTTH and optical LAN synergy enabled by virtual OLT for home, office and campus. In 45th European Conference on Optical Communication (ECOC), 2019.

[18] R. Vilalta et al., "GRPC-based SDN control and telemetry for soft-failure detection of spectral/spacial superchannels," 45th European Conference on Optical Communication (ECOC 2019), Dublin, Ireland, 2019.

[19] Tibit Communication, [Online]. Available: https://tibitcom.com/

[20] Skylane Optics, [Online]. Available: https://www.skylaneoptics.com/en/

[21] Juniper Products, [Online]. Available: https://www.juniper.net/

[22] « Données sur les installations radioélectriques de plus de 5 watts » 2017 , ANFR, Available: https://www.data.gouv.fr/fr/datasets/donnees-sur-lesinstallations-radioelectriques-de-plus-de-5-watts-1/. In French 